\title{
The impact of COVID-19 on dietary and lifestyle behaviours in a sample of Irish higher education students
}

\author{
S. Doak ${ }^{1}$, J. Kearney ${ }^{2}$, J.M. McCormack ${ }^{1}$ and L. Keaver ${ }^{1}$ \\ ${ }^{1}$ Department of Health and Nutritional Science, Institute of Technology Sligo, Sligo, Ireland and \\ ${ }^{2}$ School of Biological and Health Sciences, Technological University Dublin, Dublin, Ireland
}

The COVID-19 pandemic may have potentially impacted the diet and lifestyle behaviours of vulnerable groups like higher education students $^{(1)}$. Recent studies found that health behaviours such as diet, physical activity, sleep, and alcohol consumption changed during lockdowns affecting the physical and mental health of students ${ }^{(2,3)}$. Other studies found differing results, dependent on location, lockdown restrictions and cultural differences ${ }^{(4)}$. The purpose of this study was to examine the self-reported impact of Covid-19 on diet and lifestyle behaviours, in a sample of Irish higher education students.

Data was collected as part of a cross-sectional web-based survey, hosted by Qualtrics®XM in the 2020-2021 academic year, during varying stages of the COVID-19 pandemic. Registered full-time students, aged 18 and over, from three institutes in North-West Ireland were eligible to participate. Self-reported information was collected in relation to demographics. A similar question was asked about the impact of COVID-19, adapted from previous research ${ }^{(3)}$ relating to diet, sleep, stress, physical activity, and alcohol use: "Has the COVID-19 pandemic impacted your alcohol intake/ sleeping patterns/ stress levels/ physical activity levels/ dietary intake?". Answers were given on a similar 4-point Likert scale e.g. 'Yes - I drink more,' 'Yes - I drink less,' 'no,' and 'unsure'. SPSS was used to conduct a descriptive statistical analysis and presented as Number (\%) or Mean \pm Standard Deviation.

A total of 682 completed responses were recorded. The mean age was $24.97 \pm 8.90$ and most students were female $(n=455,66.8 \%$, $)$ in first year $(n=208,30.6 \%)$, and science students $(n=312,46.6 \%)$. When asked about the impact of COVID-19 on dietary behaviours $29.5 \%(n=195)$ reported that their diet is less healthy and $26.4 \%(n=174)$ reported eating more healthily while $37.9 \%(n=257)$ reported cooking or preparing fresh food more often and $15.8 \%(n=107)$ did not. Almost half $(n=332,48.8 \%)$ of students reported drinking less and $11.7 \%(n=80)$ consume more alcohol than usual. Sleep was negatively impacted in $39.0 \%(n=266)$ of students with only $14.1 \%(n=96)$ reporting better quality sleep. Most students $(n=411,62.1 \%)$ feel more stressed than usual, with few $(n=57$, $8.6 \%)$ reporting that they are feeling less stressed. Finally, $54.8 \%(n=372)$ of students reported being less physically active with $25.2 \%(n=171)$ reporting being more active than usual.

This research found that students attending the institutes in the North-West of Ireland were impacted by the COVID-19 pandemic, feeling more stressed, reporting poorer sleep quality, and less participation in physical activity. However dietary habits did not seem to be as negatively impacted and alcohol consumption was improved, as self-reported by students. These results indicate the selfreported impacts of a global pandemic and lockdown restrictions on health behaviours in this cohort.

\section{Acknowledgments}

The authors wish to thank Jason Quinn, Keelan Kennoy and Ryan O'Reilly from ITS Students' Union, Yvonne Roache from ITS, Colin Kearney and Victor O'Loughlin from GMIT Students' Union and Mary Hernandez from LYIT Students' Union for helping with survey promotion. In addition, the authors thank the students who participated in this study as volunteers, making the study possible.

\section{References}

1. Bertrand L, Shaw KA \& Ko J (2021) Appl Physiol Nutr Metab 46, 265-272.

2. Ingram J, Maciejewski G \& Hand CJ (2020) Front Psychol 11.

3. Du C, Zan MCH, Cho MJ et al. (2021) Clocks \& Sleep 3, 12-30.

4. Palmer K, Bschaden A \& Stroebele-Benschop N (2021) Appetite 167, 105638. 